\title{
Abordagem cirúrgica para cisto periapical de tamanho atípico em idosa: Relato de
}

\section{caso}

\author{
Surgical appproach for atypical size periopic cyst in elderly: Case report
}

Abordaje quirúrgico de un quiste periapical de tamaño atípico en una anciana: Reporte de caso

Priscilla Sarmento Pinto

ORCID: https://orcid.org/0000-0002-2376-4383 Faculdade de Odontologia de Pernambuco, Brasil

Universidade Federal de Pernambuco, Brasil E-mail: priscillasarmentop@gmail.com

Rosa Rayanne Lins de Souza

ORCID: https://orcid.org/0000-0002-9534-605X

Faculdade de Odontologia de Pernambuco, Brasil

E-mail: rosaalins27@hotmail.com

Ruan de Sousa Viana

ORCID: https://orcid.org/0000-0003-1542-4554

Faculdade de Odontologia de Pernambuco, Brasi

E-mail: ruansv@hotmail.com

Lucas Emmanuell de Morais Neves

ORCID: https://orcid.org/0000-0001-7257-3148

Hospital da Restauração, Brasil

E-mail: lucas_emmanuell@hotmail.com

Felipe Ricardo Cisneiros Brito

ORCID: https://orcid.org/0000-0001-8222-9033

Faculdade de Odontologia de Pernambuco, Brasil

E-mail: felipe.cisneiros@upe.br

Carlos Augusto Pereira do Lago

ORCID: https://orcid.org/0000-0001-9457-714X Hospital da Restauração, Brasil

E-mail: caloslago1810@hotmail.com

Greiciane Miguel de Azevedo Santos

ORCID: https://orcid.org/0000-0001-7876-3655

Faculdade de Odontologia de Pernambuco, Brasil

E-mail: greiciane_azevedo@outlook.com

Arthur Alves Thomaz de Aquino

ORCID: https://orcid.org/0000-0002-5664-015X

Faculdade de Odontologia de Pernambuco, Brasil

E-mail: arthur.aquino29@gmail.com

João Victor Souza Pinto

ORCID: https://orcid.org/0000-0003-4224-3492 Centro Universitário de João Pessoa, Brasil

E-mail: jvsp.odontologia@gmail.com

Ana Cléa Aguiar de Araujo Lima

ORCID: https://orcid.org/0000-0002-9988-7988

Centro Universitário de João Pessoa, Brasil

E-mail: anaclea.odontologia @gmail.com

Lívia Mirelle Barbosa

ORCID: https://orcid.org/0000-0002-8992-2890

Universidade de Pernambuco, Brasil

E-mail: dra.liviabarbosa@gmail.com

\begin{abstract}
Resumo
Os cistos periapicais, são formados pelo processo inflamatório do epitélio no ápice de dentes não vitais. Normalmente apresenta características de crescimento lento, assintomático e de pequena extensão. Nesse estudo, objetiva-se reportar um caso de cisto periapical com tamanho atípico, submetido à abordagem cirúrgica. Aqui, trata-se de um estudo qualitativo, descritivo, retrospectivo e realizado pela da técnica da observação direta, respeitando todos os protocolos éticos. Paciente 74 anos, feminina, compareceu ao serviço de Cirurgia e Traumatologia Buco-maxilo-facial do Hospital da Restauração Governador Paulo Guerra - Recife, encaminhada por um cirurgião-dentista após análise de exame de imagem de rotina sugestivo de lesão cística em mandíbula. Ao exame físico apresentou discreto abaulamento em cortical vestibular de corpo mandibular direito, assintomático ao toque. Através da tomografia computadorizada se constatou uma área hipodensa, unilocular, circunscrita por uma linha hiperdensa, abrangendo
\end{abstract}


região de dentes 42,43,44,e 45. Com base no exame clínico, foi estabelecida a hipótese diagnóstica de cisto periapical, confirmada após análise histopatológica. Como abordagem terapêutica optou-se por realizar sob anestesia geral a enucleação, osteotomia periférica e exodontias dos dentes envolvidos. Em 08 meses de acompanhamento não apresenta sinal de recidivas. Desse modo, fica claro a importância do correto diagnóstico, bem como a eficácia da intervenção cirúrgica como tratamento de lesão periapical de grande extensão.

Palavras-chave: Cistos maxilomandibulares; Cisto radicular; Cirurgia bucal.

\begin{abstract}
Periapical cysts are formed by the inflammatory process of the epithelium at the apex of non-vital teeth. It usually presents characteristics of slow growth, asymptomatic and of small extension. In this study, the objective is to report a case of periapical cyst with atypical size, submitted to surgical approach. Here, this is a qualitative, descriptive, retrospective study carried out using the technique of direct observation, respecting all ethical protocols. A 74-yearold female patient attended the Buco-maxillofacial Surgery and Traumatology service at Hospital da Restauração Governador Paulo Guerra - Recife, referred by a dentist after analysis of a routine image exam suggestive of a cystic lesion in the mandible. On physical examination, she presented a slight bulging in the vestibular cortex of the right mandibular body, asymptomatic to the touch. Computed tomography revealed a hypodense, unilocular area, circumscribed by a hyperdense line, covering the region of teeth lower lateral incisor, right lower cuspid (canine), right lower firt premolar and right lower second premolar. Based on the clinical examination, the diagnostic hypothesis of periapical cyst was established, confirmed after histopathological analysis. . As a therapeutic approach, it was decided to perform enucleation, peripheral osteotomy and extractions of the teeth involved under general anesthesia. After 8 months of follow-up, she shows no sign of recurrence. Thus, it is clear the importance of correct diagnosis, as well as the efficacy of surgical intervention as a treatment for large periapical lesions.
\end{abstract}

Keywords: Jaw cysts; Radicular cyst; Surgery oral.

\title{
Resumen
}

Los quistes periapicales están formados por el proceso inflamatorio del epitelio en el vértice de los dientes no vitales. Suele presentar características de crecimiento lento, asintomático y de pequeña extensión. En este estudio, el objetivo es reportar un caso de quiste periapical de tamaño atípico, sometido a abordaje quirúrgico. En este caso, se trata de un estudio cualitativo, descriptivo, retrospectivo realizado mediante la técnica de observación directa, respetando todos los protocolos éticos. Paciente femenina de 74 años que acudió al servicio de Cirugía y Traumatología Bucomaxilofacial del Hospital da Restauração Governador Paulo Guerra - Recife, remitida por un odontólogo tras el análisis de un examen de imagen de rutina sugestivo de una lesión quística en la mandíbula. Al examen físico presentaba un leve abombamiento en la corteza vestibular del cuerpo mandibular derecho, asintomático al tacto. Mediante tomografía computarizada se encontró un área hipodensa, unilocular, circunscrita por una línea hiperdensa, que recubre la región de los dientes 42,43,44 y 45. Con base en el examen clínico se estableció la hipótesis diagnóstica de quiste periapical, confirmada tras histopatología. análisis. Como abordaje terapéutico, se decidió realizar enucleación, osteotomía periférica y extracciones de los dientes involucrados bajo anestesia general. En 08 meses de seguimiento, no hay signos de recurrencia. Así, queda clara la importancia de un correcto diagnóstico, así como la eficacia de la intervención quirúrgica como tratamiento de grandes lesiones periapicales.

Palabras clave: Quistes maxilomandibulares; Quiste radicular; Cirugía bucal.

\section{Introdução}

Cistos são definidos como uma cavidade patológica, contendo conteúdo fluido, semifluido ou gasoso, possuindo um revestimento de tecido epitelial originado da proliferação dos restos de Malassez. Tais entidades podem ser classificadas como odontogênicos e não odontogênicos, com base no tecido de origem (Resente et al., 2017; Kolari et al., 2019; Talukdar et al., 2020; Tian et al., 2019; Noda et al., 2019; Nik et al., 2020). Dentre os cistos odontogênicos, o cisto periapical é considerado o mais frequente, seguido do cisto dentígero (Botelho et al., 2017; Noda et al., 2019; Talukdar et al., 2020; Tian et al., 2019; Rajendra 2020).

Os ossos com a maior prevalência de acometimento císticos no corpo humano são os maxilares, devido apresentarem quantidades abundante de restos epiteliais (Kolari et al., 2019; Pereira et al., 2019). Dentre as regiões dos maxilares, a área perirradicular é acometida em aproximadamente 90\% dos casos (Pitcher et al., 2019; Nik et al., 2020).

Por meio de estudos epidemiológicos pode-se traçar o perfil dos pacientes acometidos pelos cistos periapical como individuo do sexo masculino entre a terceira a sexta década de vida (Comim et al., 2017; Noda et al., 2019; Rajendra 2020). 
Apresenta-se frequentemente de forma assintomático, com exceção para os casos em que exista uma resposta inflamatória aguda (Botelho et al., 2017; Comim et al., 2017; Talukdar et al., 2020). O crescimento geralmente é lento e apresenta ampla variação de tamanho, entre 0,5 a 1,5 cm (Bilodeau and Collins 2017; Resende et al., 2017). Com o crescimento, podem ocorrer mobilidade e deslocamento dos elementos dentários adjacentes (Kolari et al., 2019; Rajendra 2020)

Comumente, a lesão é descoberta por meio de exame radiográfico de rotina (Resende et al., 2017; Kolari et al., 2019). Dentre os exames de imagem, a tomografia computadorizada permite a avaliação tridimensional das lesões perirradiculares, sendo considerada uma ferramenta útil na avaliação pré-cirurgica, determinando seus limites e possiveis conteúdos, bem como auxiliando na abordagem clínica, no entanto não apresenta precissão para a definição de diagnóstico definitivo, sendo esse obtido por meio de estudo histopatológico (Pitcher et al., 2017).

O exame histopatológico é fundamental para concluir um diagnóstico, descartando outras possíveis lesões. Como diagnóstico diferencial para caso de cisto periapical, destaca-se o ceratocisto odontogênico, lesão central de células gigantes, ameloblastoma e outros cistos e tumores odontogênico (Resende et al., 2017).

Diversos tratamentos podem ser indicados para esta lesão, sendo frequentemente submetidos a abordagens terapeuticas cirurgicas, principalmente nos casos em que o tratamento endodôntico não resultar em reparo tecidual. Entre os tratamentos cirurgicos descritos na literatura, pode citar a enucleação total e a marsupialização ou descompressão (Comim et al., 2017; Kolari et al., 2019; Tian et al., 2019; Nik et al., 2020).

Este estudo tem por objetivo descrever um caso clínico com abordagem terapêutica cirúrgica de cisto periapical de tamanho atípico em mandíbula de paciente com idade avançada.

\section{Metodologia}

Todas as informações do paciente em questão foram colhidas através do acesso direto ao prontuário e exames de imagem, a fim de descrevê-los sistematicamente. Tomando como base os princípios éticos propostos por Helsinki, o paciente consentiu com a divulgação do seu caso com finalidade acadêmica por meio da assinatura do Termo de Consentimento Livre e Esclarecido. Esse estudo é qualitativo, descritivo, retrospectivo e realizado pela da técnica da observação direta (Pereira et al., 2018) e os dados do paciente foram avaliados após diagnóstico.

\section{Relato de Raso}

Paciente 74 anos, feminina, leucoderma, sem alterações sistêmicas, compareceu ao serviço de Cirurgia e Traumatologia Buco-maxilo-facial do Hospital Da Restauração Governador Paulo Guerra - Recife, encaminhada por um cirurgião-dentista após análise de exame de imagem de rotina sugestivo de lesão cística em mandíbula. Paciente sem queixas dignas de nota no momento da anamnese.

Ao exame físico apresentou discreto abaulamento em cortical vestibular de corpo mandibular direito, assintomatica e oclusão pouco funcional. Por meio de exame de imagem, do tipo tomografia computadorizada de feixe cônico (Cone Bean), apresentou uma área hipodensa, unilocular, circunscrita por uma linha hiperdensa, extensa, abrangendo região correspondente ao alvéolo do dente 42, e ao periápice dos dentes 43, 44 e 45, com perda de lâmina dura, sem deslocamento dentário e sem reabsorção radicular (Figura 1). Os elementos dentários 42 e 44 responderam ao teste de sensibilidade pulpar, diferente dos dentes 43 e 45 que apresentavam condutos já tratados endodonticamente (Figura 1). 
Figura 1: Exame de imagem do tipo tomografia Cone Bean apresentando área hipodensa, bem delimitada, em região de corpo mandibular, sugestiva de lesão cística em mandíbula.

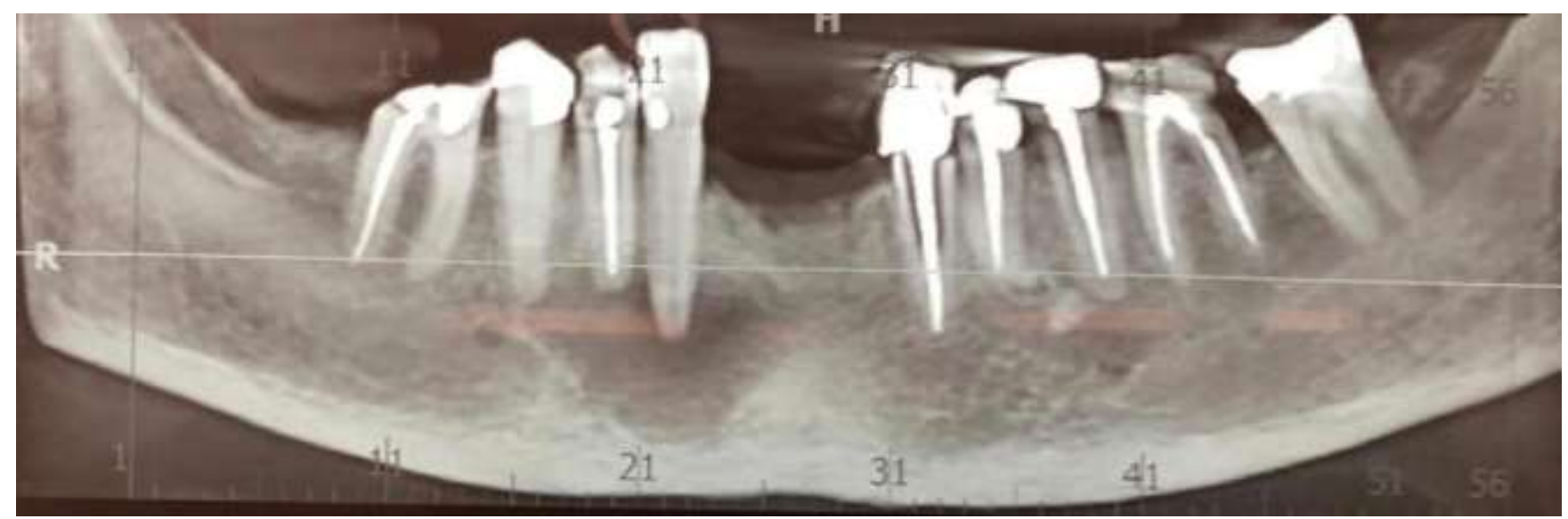

Fonte: Autores.

Com base no exame físico e de imagem, foi estabelecido a hipótese diagnóstica de cisto periapical, optando assim pela abordagem cirurgica da lesão sob anestesia geral, por meio de acesso intra oral (Figura 2A), para enucleação (Figura 2B) e osteotomia periférica associada a exodontia dos dentes envolvidos (Figura 2C). Foi realizada limpeza copiosa e sutura por planos, evitando devidas complicações (Figura 2D).

Figura 2.A: Abordagem cirurgica da lesão sob anestesia geral, por meio de acesso intra oral. Fig 3.B: Enucleação cística. Fig 2.C: Osteotomia periférica associada a exodontia dos dentes envolvidos. Fig 2.D: Limpeza copiosa e sutura por planos.
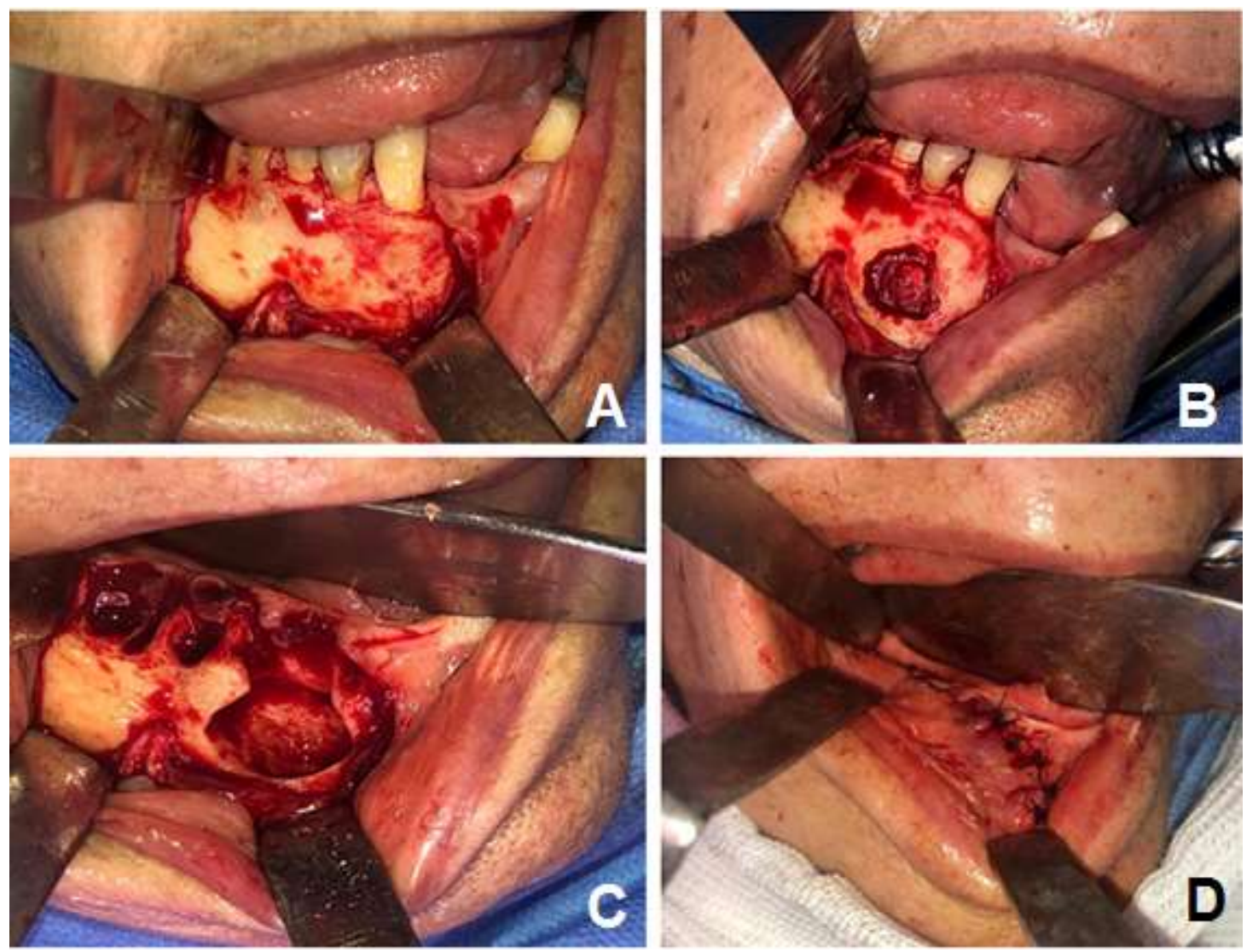

Fonte: Autores. 
A peça foi enviada para análise histopatológica, confirmando o diagnóstico de lesão compatível com cisto periapical. A paciente está sob acompanhamento há oito meses pós-operatorio sem sinal de recidiva e com o reparo ósseo da região perirradicular adequado (Figura 3).

Figura 3: Tomografia computadorizada de acompanhamento de oito meses pós-operatorio sem sinal de recidiva e com o reparo ósseo da região perirradicular adequado.

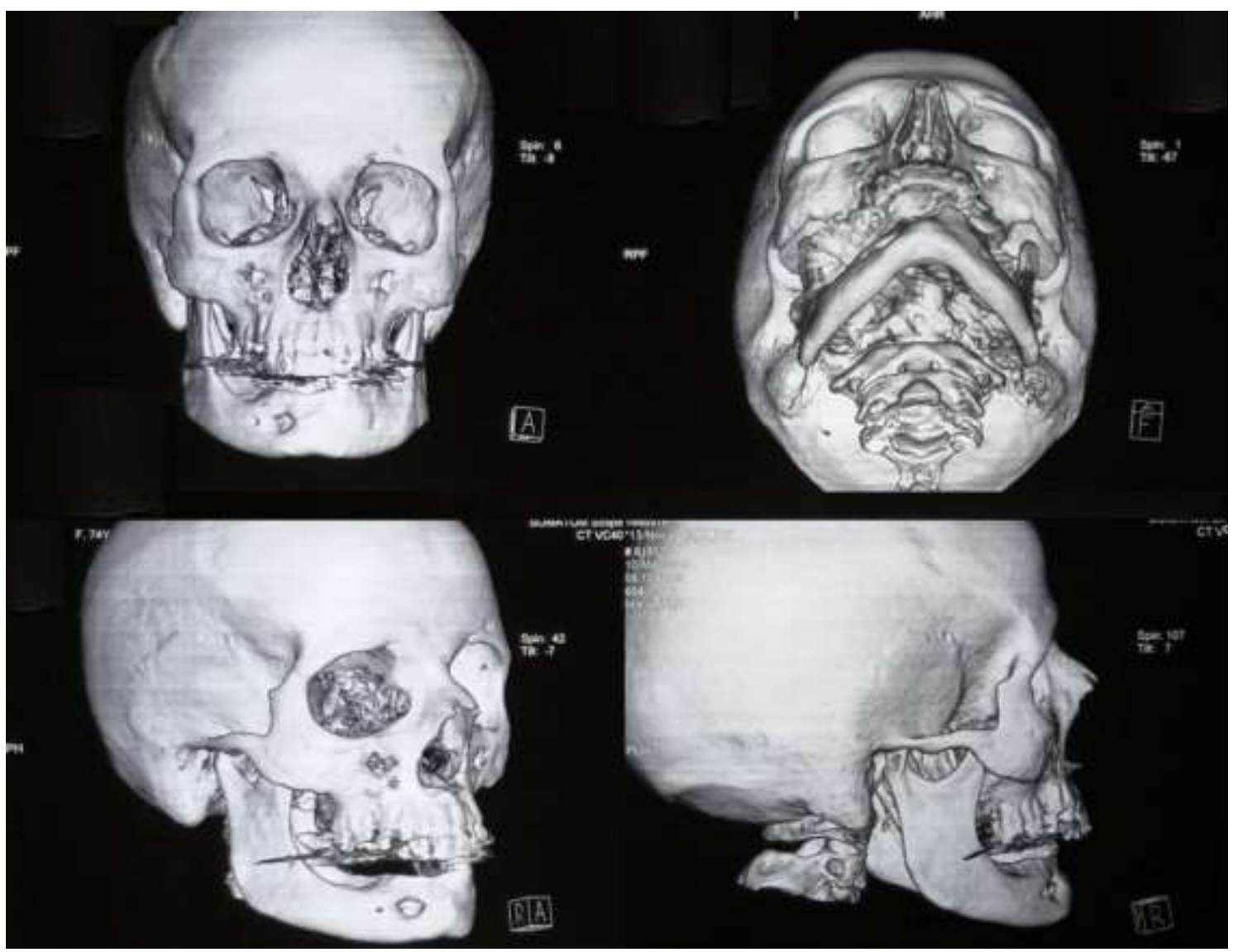

Fonte: Autores.

\section{Discussão}

Cistos periapicais, também denominado como cisto radicular, são alterações que se originam dos restos epiteliais de Malassez no ligamento periodontal que envolve um dente com aspecto inflamatório periapical crônico (Bilodeau \& Collins 2017; Resende et al., 2017; Nik et al., 2020).

Tal cisto é considerado o mais comum dos cistos odontogênicos inflamatórios em uma frequência de $52 \%$ a $68 \%$, com maior acometimento em adultos jovens (Botelho et al., 2017; Comim et al., 2017; Tavares et al., 2017; Lee et al., 2019; Noda et al., 2019; Pereira et al., 2019). No entanto, alguns estudos apresentam tal lesão como a segunda mais prevalente, assim como descrito no estudo epidemiológico realizado por Lee et al. (2019), o cisto dentígero foi o mais frequente, seguido do cisto periapical, em pacientes com idade média de 37,6 anos no momento da cirurgia, não corroborando com o caso aqui descrito, a paciente apresentava 74 anos.

Comim et al. (2017) descreve em seu estudo uma ordem de acometimento nas regiões dos maxilares, sendo a porção anterior da maxila mais prevalente, seguido de região posterior da maxila, posterior da mandíbula e, por fim, a região anterior da mandíbula. Pereira et al. (2019) descreve o acometimento em região apical bem como nas superfícies laterais das raízes, 
devido aos canais acessórios. No caso aqui descrito, delimita-se a área cística entre a porção anterior e posterior mandibular, com envolvimento dos ápices dentários.

De acordo com a extensão da lesão, Comim et al., (2017) descreve que a quantidade de osso cortical pode influenciar a velocidade do desenvolvimento cístico, e esse fator ser determinante para maior frequência de lesões de grandes diâmetros acometerem mais a maxila do que a mandíbula.

As características clínicas são bem estabelecidas na literatura de forma assintomática, de pequena extensão, atingindo até $1,5 \mathrm{~cm}$, com o elemento dentário envolvido no processo de inflamação apresentando resposta pulpar negativa frente ao teste térmico e elétrico. Os dentes envolvidos bem como adjacentes podem apresentar mobilidade e deslocamento devido ao crescimento cístico (Bilodeau \& Collins 2017; Botelho et al., 2017; Pereira et al., 2019). No presente caso, notou-se uma lesão assintomática de grande extensão (15 milímetros nas suas maiores extensões), com lesão pulpar envolvida e sem mobilidade e deslocamento dentário.

Segundo Bilodeau et al. (2017), Noda et al. (2019) e Nik et al. (2020), o dente envolvido pelo cisto pode apresentar-se com história de trauma local ou cariado. No caso aqui descrito, os dentes envolvidos apresentavam tecidos cariados, bem como tratamento endodôntico não eficaz.

Radiograficamente, a descrição clássica dos cistos periapicais apresenta-se como uma área radiolúcida circular ou oval, circunscrita por uma linha radiopaca bem definida e envolvendo os ápices dentários infectado, correspondendo entre 7\% a 54\% das imagens radiolúcidas periapicais (Bilodeau \& Collins 2017; Botelho et al., 2017; Comim et al., 2017; Resende et al., 2017; Noda et al., 2019; Talukdar et al., 2020).

Segundo Comim et al. (2017) os cistos periapicais apresentam dificuldade no diagnóstico devido suas características clínicas e de imagem apresentarem semelhanças com outras lesões apicais. Pereira et al., (2019) descreve os tumores ósseos, granuloma periapical e outros cistos devem ser considerados como diagnóstico diferencial. Por tal motivo, a análise histopatológica é considerada o padrão ouro do diagnóstico de lesões perirradiculares, sendo então realizada no presente caso.

No entanto apesar da avaliação histopatológica ser considerada um teste diagnóstico definitivo, segundo Pitcher et al. (2017) a mesma apresenta como fator negativo a inviabilização da avaliação pré-operatória das lesões devido ao fato de necessitar de excisão cirúrgica.

Segundo Kolari et al. (2019) a escolha da abordagem terapeutica deve levar em consideração os fatores como extensão da lesão, aspecticos clínicos e relação com estruturas adjacentes. Pereira et al. (2019) destaca a importância de conhecer o estado de saúde geral do paciente, por meio de uma história médica minuciosa. No caso aqui relatado, uma avaliação detalhada descartou alterações sistêmicas, possibilitando a abordagem terapêutica cirúrgica.

As opções terapêuticas para as lesões císticas periapicais variam desde o tratamento não cirúrgico ao tratamento cirúrgico, ou associação de ambos. Resende et al. (2019) e Nik et al. (2020) descrevem que a abordagem cirúrgica é indicada para lesões extensas, maiores de $1,5 \mathrm{~cm}$ no seu maior diâmetro, bem como para casos em que não é viável o tratamento endodôntico convencional.

No caso aqui descrito, a paciente já havia passado por tratamentos endodônticos previamente, não apresentando sucesso e, portanto, optou-se por realizar procedimento cirúrgico de enucleação, curetagem e exodontia de dentes envolvidos, já que a mesma encontrava-se colaborativa e sistemicamente apta para tal procedimento. Comim et al. (2017) descreve que a exodontia, quando escolhida como forma de tratamento, deve sempre ser associada a curetagem óssea periapical.

Os cistos de origem inflamatória demonstram baixa taxa de recidiva após tratamento cirúrgico. No entanto, segundo Resende et al. (2017) e Tian et al. (2019) após abordagem cirúrgica pode ocorrer formação de cicatrizes fibrosas em vez de neoformação óssea. No caso aqui descrito, a paciente encontra-se no oitavo mês pós-operatório, com reparo ósseo adequado e até o momento não apresenta quaisquer sinais de recidiva. 


\section{Conclusão}

Diante o caso aqui exposto de uma paciente com idade avançada submetida a tratamento cirúrgico para remoção de cisto periapical de tamanho atípico, nota-se que através de um correto e minucioso diagnóstico e plano de tratamento, os cistos periapicais de grande extensão tratados por meio cirúrgicos apresentam prognóstico favorável, promovendo o reparo da região perirradicular.

\section{Referências}

Bilodeau, E. A., \& Collins, B. M. (2017). Odontogenic cysts and neoplasms. Surgical pathology clinics, 10(1), $177-222$.

Botelho, P. M., Barbosa, D. D. A., Costa, L. C., Gonzalez, J. L., \& Pimentel, R. M. (2018). Enucleação de lesão perirradicular (cisto periapical)-relato de caso. Ciência Atual-Revista Científica Multidisciplinar do Centro Universitário São José, 9(1).

Comim, L., Durant, V., De Carli, J. P., Rinaldi, I., \& Linden, M. S. S. (2017). Cisto periapical de grandes proporções na região anterior da maxila. Relato de caso. Rev. Salusvita (Online), 501-508.

Dos Santos Pereira, R., de Souza Guimaraes, V. C., Timóteo, C. A., Homsi, N., da Rocha Jr, H. V., \& Hochuli-Vieira, E. (2014). Internal maxillary artery pseudoaneurysm subsequent gunshot wound in a teenager. The Journal of craniofacial surgery, 25(3), 1125-1126.

Kolari, V., Rao, H. A., \& Thomas, T. (2019). Maxillary and mandibular unusually large radicular cyst: a rare case report. National journal of maxillofacial surgery, 10(2), 270.

Lee, H., Lee, S. J., \& Seo, B. M. (2019). Investigation of postoperative complications of intrabony cystic lesions in the oral and maxillofacial region. Journal of Oral and Maxillofacial Surgery, 77(9), 1823-1831.

Nik Abdul Ghani, N. R., Abdul Hamid, N. F., \& Karobari, M. I. (2020). Tunnel'radicular cyst and its management with root canal treatment and periapical surgery: A case report. Clinical case reports, 8(8), 1387-1391.

Noda, A., Abe, M., Shinozaki-Ushiku, A., Ohata, Y., Zong, L., Abe, T., \& Hoshi, K. (2019). A bilocular radicular cyst in the mandible with tooth structure components inside. Case reports in dentistry, 2019.

Pitcher, B., Alaqla, A., Noujeim, M., Wealleans, J. A., Kotsakis, G., \& Chrepa, V. (2017). Binary decision trees for preoperative periapical cyst screening using cone-beam computed tomography. Journal of endodontics, 43(3), 383-388.

Rajendra Santosh AB. (2020). Odontogenic Cysts. Dental clinics of North America, 64(1):105-19.

Resende, M. A. P., Assis, N. M. S. P., Sette-Dias, A. C., de Aguiar, E. G., \& Sotto-Maior, B. S. (2017). Tratamento cirúrgico e conservador de cisto periapical de grande proporção: relato de caso. HU Revista, 43(2), 191-196.

Ribas Pereira, J. A., Caciatori Garcia, K., Verbicaro, T., Nascimento Meger, M., Cotait de Lucas Corso, P. F., \& Scariot, R. (2019). Tratamento cirúrgico de cisto periapical inflamatório de grande extensão em maxila: relato de caso. RSBO: Revista Sul-Brasileira de Odontologia, 16(1).

Talukdar, M., Kumar, A., Goenka, S., Mahajani, M., Ambhore, M. P., \& Tattu, V. D. (2020). Management of radicular cyst in deciduous molar: A case report. Journal of family medicine and primary care, 9(2), 1222.

Tavares, D. P., \& Rodrigues, J. T. (2017). Clinical and radiological analysis of a series of periapical cysts and periapical granulomas diagnosed in a Brazilian population. Journal of clinical and experimental dentistry, 9(1), e129.

Tian, F. C., Bergeron, B. E., Kalathingal, S., Morris, M., Wang, X. Y., Niu, L. N., \& Tay, F. R. (2019). Management of large radicular lesions using decompression: a case series and review of the literature. Journal of endodontics, 45(5), 651-659. 